\title{
One-Dimensional Standing and Sitting Interface Based on Relative Distances
}

\author{
Geunho Lee ${ }^{\mathrm{a},}{ }^{,}$, Yuki Fujio ${ }^{\mathrm{a}}$, Go Yamako ${ }^{\mathrm{a}}$, Hiroki Tamura ${ }^{\mathrm{a}}$, Etsuo Chosa ${ }^{\mathrm{b}}$ \\ ${ }^{a}$ Faculty of Engineering, University of Miyazaki, 1-1 Gakuen, Miyazaki, Kibana-dai-nishi 889-2192, Japan \\ ${ }^{\mathrm{b}}$ Faculty of Medicine, University of Miyazaki, 5200 Kihara, Miyazaki, Kiyotake 889-1692, Japan \\ *Corresponding Author: geunho@cc.miyazaki-u.ac.jp
}

\begin{abstract}
When developing an assist device for elderly people, an easy-to-learn and simple-to-use interface system capable of responding to complex and diverse environments is of particular importance. Since the assist device is expected to employ in various kinds of public places, more than anything, protection of personal data is its fundamental requirements. For this purpose, our paper tackles a problem of designing an interface between elderly users and assist devices helping them stand up and sit down. As a solution approach, the paper introduces a one-dimensional distance-based interface, allowing to recognize the 3D standing and sitting behaviors, without requiring additional sensor inputs such as visual data.
\end{abstract}

Keywords: proximity sensor, relative distance, standing and sitting behaviors, user interface, welfare robotics.

\section{Introduction}

Recently, buttons [1] or levers [2] as one type of user interfaces have been widely used in standing and sitting assist devices such as lift chairs and automatic lifting commodes. In detail, the interfaces used to support standing and sitting behaviors can be categorized into the following three types. The first interface uses levers, buttons, joystick, and pressure sensors. These types of interface, which are primarily used to operate lift chairs, i.e., chairs that move vertically up/down, are cost-efficient and user-friendly. These interfaces are a simple way to intuitionally define target operations like an on-off control. If more functions are added to the assist devices, more numbers of buttons are needed. The mental workload involved might increase and could potentially cause confusion for the elderly users, accordingly resulting in increasing the likelihood of an accident. Another type of interface uses image data obtained from a camera. This is effective because a large amount of data can be obtained without touching the camera; however, the image quality gets affected by light conditions and surrounding environment. In addition, privacy issues and cost required for processing large volumes of information must be considered. The third type of interface, which is primarily used in wearable support devices, uses muscle potential and frequency analysis to detect changes in fatigue caused by muscle movement. Wearable support devices that rely on muscle potential are very effective; however, considering the effort required to wear such devices and their size, they are not suitable for everyday use.

When designing and developing assist devices for the elderly, a simple, easy interface is of particular importance [3-4]. Furthermore, the interface should protect their own privacy in order to utilize public places. Unlike existing studies, our study aims to propose an interface scheme to recognize standing and sitting using only distance data. The proposed interface allows to estimate an operating state based on distance data obtained from a measurement sensor and to support appropriate behaviors relative to the estimated state. Based on the considerations above, the following problem is addressed: "How to represent the behavioral intents of the user's standing and sitting without the invasion of privacy?" Our solution for the problem allows a distancebased interface by using only a proximity sensor. In this study, we conducted an experiment using the proposed interface method to verify its effectiveness.

\section{Distance-Based Interface}

\subsection{Definitions of standing and sitting}

To define standing and sitting behaviors, a rigid bar model is designed. To begin, we assume a rigid-body link 
model, as shown in Fig. 1. Here, the link model is divided into the following three parts with the body facing toward the right: (from the bottom) 'c': foot to knee, 'b': knee to pelvis, and ' $a$ ': pelvis to neck. Moreover, the standing and the sitting states are denoted as $A$ and $A^{\prime}$, respectively. In this model, it is assumed that the standing and the sitting operations are only in the anteroposterior (front to back) and vertical (top to bottom) directions, while 'c' does not move.

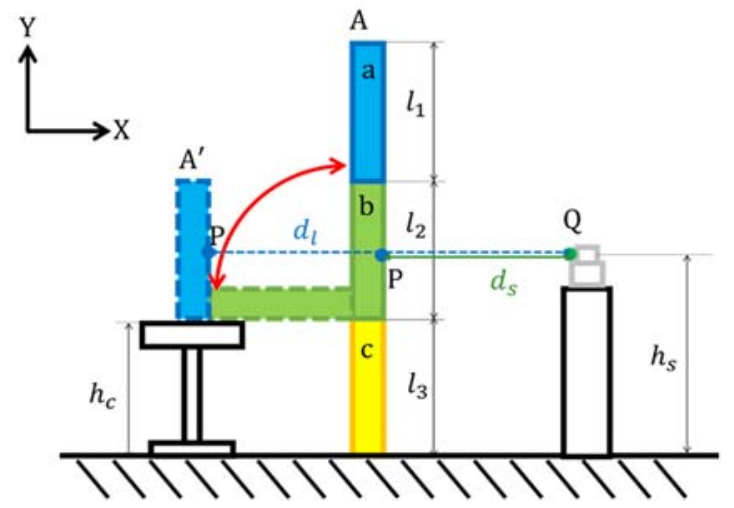

Fig. 1. Illustration of geometric definitions and notations for standing and sitting behavior.

Next, the $Y$-axis is the vertical direction from the floor to the top and the $X$-axis is the horizontal direction to the right. The length of each link is defined as $(a, b, c)=\left(l_{1}, l_{2}\right.$, $l_{3}$ ). In the $A$ state, 'a', 'b', and 'c' are oriented along the same line. In the $A^{\prime}$ state, 'a', 'b', and 'c' are oriented at right angles to each other. When the location of a proximity sensor is defined as a point $Q$, the height from the floor to $Q$ is $h_{s}$. Similarly, the height from the floor to chair is defined as $h_{c}$. In addition, the point of the rigid bar model that bisect the line stretching from $h_{s}$ in the horizontal direction is defined as $P$. Here, $P$ only moves along $X$ axis direction when the $A$ state changes to the $A^{\prime}$ state and vice versa. The distance between points $Q$ and $P$, i.e., the distance from $Q$ to the subject in the $A$ state, is denoted $d_{s}$, and the distance between points $Q$ and $P$ in the $A^{\prime}$ is denoted $d_{l}$, which expresses the state of the subject based on distance changes.

\subsection{Observation model}

We focus on the knee movement to recognize the standing, sitting, and transitional state between the standing and sitting states. As shown in Fig. 2, it is possible to approximate the standing and sitting behavior according to the rotation movement of the link $b$, which is centered around the knee. Based on the previously described conditions and under the assumption that the link $b$ will not move to the left or right, we consider a 2-D horizontal movement along the $X-Y$ plane. In addition, the circular motion during standing or sitting forms a fan shape at an angle $\theta$. The movement of this fan shape can be considered as a reciprocating motion between $P$ and $Q$. Since this reciprocating motion can be expressed as a distance difference between a fixed sensor and the subject, the standing and the sitting state is considered to be recognizable by measuring the difference change in this distance.

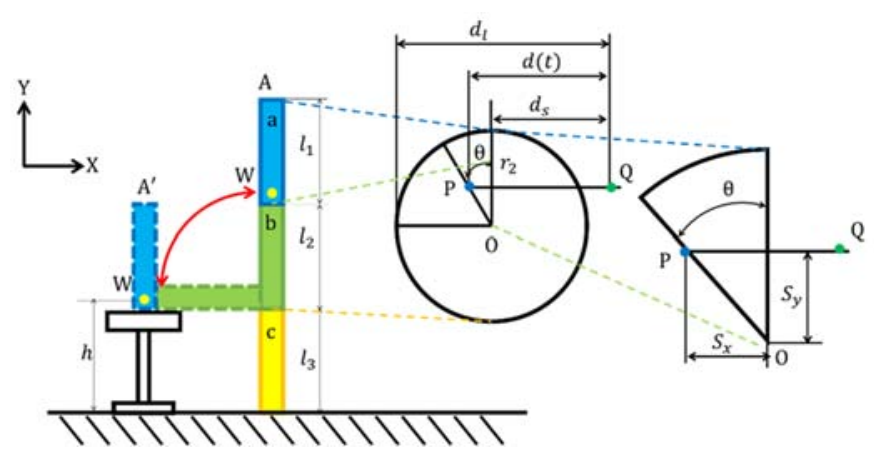

Fig. 2. Illustration of observation model based on the

Gudermannian function for standing/sitting behaviors.

From the above conditions, it is possible to approximate the standing/sitting behaviors in terms of the 2-D rotational movement of radius $r$ centering a point $O$ on the knee. In Fig. 2, this rotation is represented as the fan-shaped movement in angle $\theta\left(0^{\circ} \leq \theta \leq 90^{\circ}\right)$ by $O P$ and $O Q$. Based on the geometric consideration, we first obtain the $X$ coordinates of $O P$ from the distance between the sensor and a subject. Next, the $Y$ coordinates along the fan-shape trajectory is calculated by using the movement distance in the $Y$ direction. Consequently, the rotational motion can be considered as the one-dimensional reciprocating motion.

Furthermore, it is estimated that there will be gradual deceleration from the beginning motion to the end motion in the standing behaviors. It is possible to express the behavior as the exponential movement of angle $\theta$. Therefore, we focus on using the well-known Gudermannian function. The value of this function corresponds to the geographical latitude expressed in the Mercator projection, which can be used to calculate the distance from the equator to a latitude line on a world map. By using the Gudermannian function, a formula in which the rotation is converted to linear motion can be expressed as follows.

$$
\begin{aligned}
& d(t) \\
& =\left\{\begin{array}{l}
\frac{2}{\pi}\left(d_{l}-d_{s}\right) \tan ^{-1}(\exp (k t+l))+d_{s} \quad \text { (standing) } \\
\frac{2}{\pi}\left(d_{l}-d_{s}\right) \tan ^{-1}(\exp (-k t-l))+d_{s}
\end{array}\right. \text { (sitting) }
\end{aligned}
$$




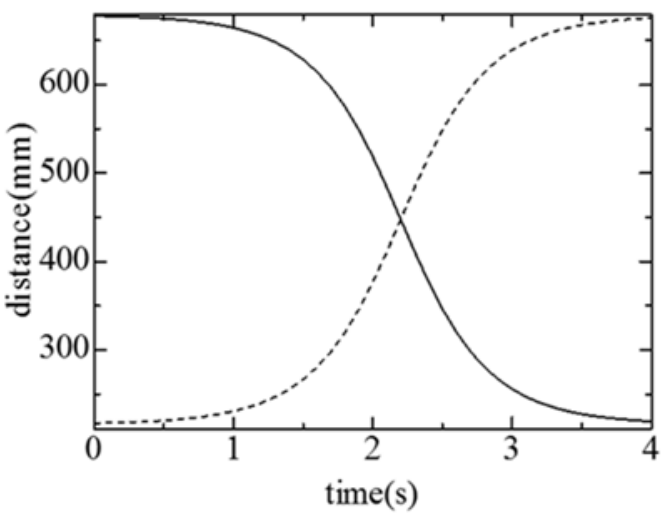

Fig. 3. Trajectories depicted by the Eq. (1) for standing and sitting behaviors, where the sold line and the dotted line indicate standing and sitting behaviors respectively.

The above formula indicates the distance change $d(t)$ between the subject and the sensor at time $t$ during standing or sitting. Specifically, the behavioral speed can be changed by modifying coefficients $k$ and $l$. The observation model designed using the Gudermannian function is shown in Fig. 3. A graph showing the mean of the distance data measured 10 times while standing and sitting is plotted in Fig. 4.

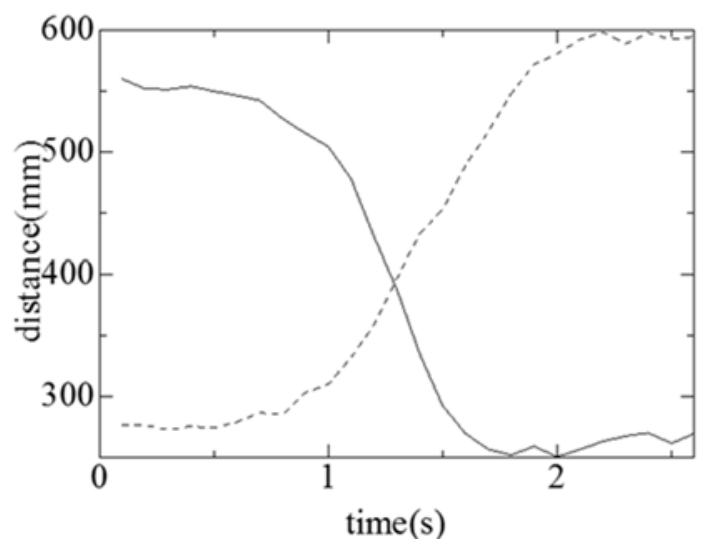

Fig. 4. Measurement results for standing-up (solid line) and sitting-down (dotted line) behaviors.

From these results, it could be confirmed that the actual distance data obtained during standing and/or sitting behaviors was similar to an observation model adjusting a measurement situation and these coefficients $k$ and $l$. As a result, we can measure $d(t)$ when standing and/or sitting, and then by using (1), determine the state of the standing or sitting behaviors.

\section{Experimental Results and Discussion}

The experiments for the effectiveness of the proposed distance-based interface by using the URG-04LX-UG01 laser range finder (LRF) were performed to examine whether the proposed interface continuously gauged distances when a subject is standing or sitting. Here, the LRF was placed on the height from the ground in the same knee of a subject participated. As shown in Fig. 1 (or Fig. 5), the LRF measured distances to one lower limb with respect to the coronal plane. Specifically, the distance indicate relative differences between the LRF and one spot in the lower limb of a subject since individual layers of the LRF at each firing time do not corresponds to the same spot while standing and sitting. From the results, it was observed that the interface allowed to measure the relative distances to the lower limb according to the behaviors.

To demonstrate the feasibility of the interface, the second experiments were conducted by 30 subjects. Before participating in our experiments, written informed consent for the publication of this study and any accompanying images was obtained from the subjects involved in all experiments. The experiments for the feasibility evaluation were performed by 30 subjects (male: 12, female: 18, age: 31-84 years, height: $142-178 \mathrm{~cm}$ ). A breakdown of the subjects' ages is: 3 persons in their thirties and 3 persons in their forties, 7 persons in their fifties, 8 persons in their sixties, 8 persons in their seventies, and 1person in his eighties. Six persons over 75 years old (male: 2, female: 4, age: $75-83$ years, height: $142-164 \mathrm{~cm}$ ) account for about $20 \%$ of all subjects.

Before all experiments, we briefly explained the measurement process and demonstrated the process. In detail, the subjects were asked to stand up according to our direction and then after 5 seconds, to sit down on a chair. Fig. 5 plots the variation data of the measurements for the standing-up and sitting-down behaviors performed by individual subjects. In these graphs, the index in the vertical axis means the distances to the lower limb of the subjects. Despite the variations of the data according to individuals, we empirically learned that the changes of relative distances were similar to the data trends in Fig. 3.

From the experiments above, the following three results are summarized. First, by using the measurement device, the relative distances could be measured according to the standing-up and sitting-down behaviors of individual subjects. Secondly, the proposed measurement scheme allowed to detect individual behaviors according to time. 
Thirdly, in spite of the use of the LRF, the measurement scheme could be discriminate between standing and sitting behaviors. From these analyses, we confirmed that our study is very valuable for the interface development of a lowerlimb assist device toward helping the potential users mentioned in Section 2. Furthermore, we expect that the scheme not requiring any visual systems can be applied to bathing, toilet and other aspects of their daily hygiene routine. As our future studies, we are going to deal with the accuracy issue of the measurement scheme under real situations and realize an interface system based on the proposed measurement scheme.

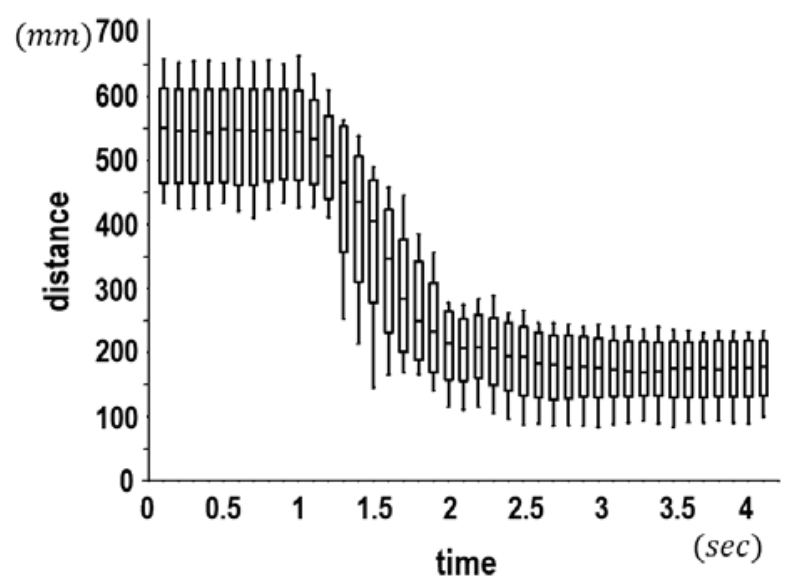

(a) standing behaviors

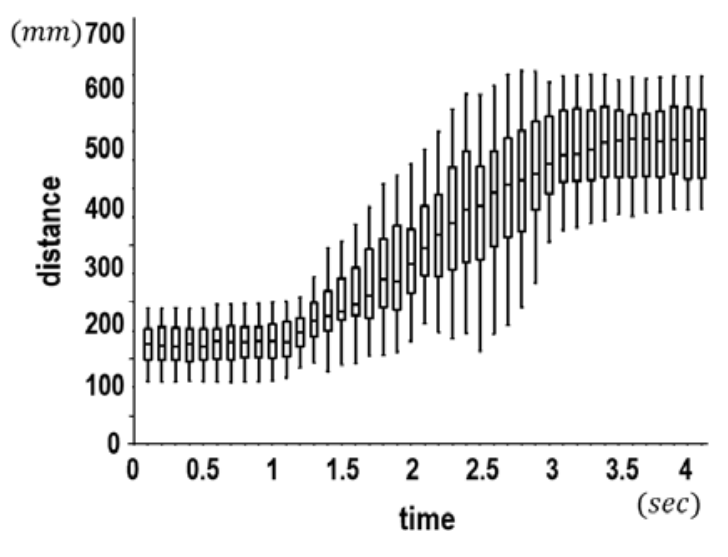

(b) sitting behaviors

Fig. 6. Statistical analysis results of 10 trials over the distance measurements of all subjects.

\section{Conclusions}

The distance-based interface for standing and sitting behaviors by only using the LRF as one proximity sensor was proposed. Specifically, the interface allowed to determine between standing behaviors and sitting ones, not requiring visual sensors such as cameras. To demonstrate the effectiveness of the interface, measurement experiments were performed. From the experiments shown in Section 3, the following three results are summarized. First, by using the interface, the relative distances could be measured according to the standing and sitting behaviors of individual subjects. Secondly, the proposed interface allowed to detect individual behaviors according to time. Thirdly, in spite of the use of the LRF, the measurement scheme could be discriminate between standing and sitting behaviors. From these analyses, we confirmed that our study is very valuable for the interface development of a lower-limb assist device. Furthermore, we expect that the interface not requiring any visual systems can be applied to bathing, toilet and other aspects of their daily routine. As our future studies, we are going to deal with the accuracy issue of the interface under real situations.

\section{Acknowledgment}

This work is partially supported by the Research Grant awarded by the Mitsubishi Foundation.

\section{References}

(1) H.-M. Gross, C. Schroeter, S. Mueller, M. Volkhardt, E. Einhorn, A. Bley, C. Martin, T. Langner, and M. Merten : "Progress in developing a socially assistive mobile home robot companion for the elderly with mild cognitive impairment", Proc. IEEE/RSJ Int. Conf. Intelligent Robots and Systems, pp. 2430-2437, 2011

(2) A.F. Neto, J.A. Gallego, E. Rocon, J.L. Pons, and R. Ceres : "Control and path planning of a walk-assist robot using differential flatness", BioMedical Engineering, Vol. 9, No. 37, pp. 1-16, 2010

(3) T. Ohnuma, G. Lee, and N.-Y. Chong: "Development of JARoW-II active robotic walker reflecting pelvic movements while walking", Journal of Intelligent Service Robotics, Vol. 10, No. 2, pp. 95-107, 2017

(4) G. Lee, T. Ohnuma, N.-Y. Chong, and S.-G. Lee : "Walking intent based movement control for JAIST active robotic walker", IEEE Trans. Systems, Man and Cybernetics: Systems, Vol. 44, No. 5, pp. 665-672, 2014 